\title{
Target-Specific Factors Regulate the Formation of Glutamatergic Transmitter Release Sites in Cultured Neocortical Neurons
}

\author{
Ralf Mohrmann, Markus Werner, Hanns Hatt, and Kurt Gottmann \\ Lehrstuhl für Zellphysiologie, Ruhr-Universität Bochum, D-44780 Bochum, Germany
}

Synapse formation in the mammalian CNS is thought to involve specific target recognition processes between presynaptic and postsynaptic neurons leading to the establishment of defined neuronal circuits. To study the role of target neuron-specific factors in synaptogenesis, we used cocultures of presynaptic explants and dissociated target neurons from rat neocortex, which enabled us to selectively vary the postsynaptic target neurons. Cocultures containing target neurons that were obtained early during development [embryonic day 16 (E16)] were compared to cocultures containing target neurons that were obtained at a later embryonic stage (E19).

Postsynaptic currents (PSCs) were evoked in target neurons by maximal extracellular stimulation in the presynaptic explant. The mean amplitudes of AMPA and NMDA receptor-mediated PSCs were sixfold reduced in E16 target neurons, whereas the mean amplitudes of GABA $_{A}$ receptor-mediated PSCs did not differ between E16 and E19 target neurons. This reduction was

During development, the highly specific synaptic connectivity of the CNS is established by a sequence of cellular and molecular processes including axonal guidance, recognition of postsynaptic targets, synaptic differentiation, and activity-dependent synaptic stabilization and elimination (for review, see Goodman and Shatz, 1993). Axonal pathfinding by neuronal growth cones is based on the balance of attractive and repulsive cues that have been identified in several cases at the molecular level (for review, see Tessier-Lavigne and Goodman, 1996). Although growth cone guidance restricts the choice of target cells to a certain CNS region, specific target cell recognition among several potential synaptic partners has to be accomplished by the presynaptic neuron at the target site. In comparison to the molecular mechanisms of axonal guidance, these target recognition processes are still poorly understood.

Current knowledge on molecular target recognition involved in synaptogenesis mainly emerged from studies at larval neuromuscular synapses in Drosophila, because in this model system the highly stereotyped motor innervation pattern facilitates experimental analysis (Keshishian et al., 1996). In Drosophila, several factors involved in target recognition have recently been identified at the molecular level by genetic analysis (Chiba et al., 1995;

\footnotetext{
Received Jan. 21, 1999; revised Sept. 1, 1999; accepted Sept. 2, 1999.

This work was supported by grants from the Deutsche Forschungsgemeinschaft. We thank Dr. V. Le $\beta$ mann for valuable comments on this manuscript and Dr. P. Wahle for the kind gift of GAD-65 antibody. We further thank H. Jung and H. Bartel for excellent technical assistance.

Correspondence should be addressed to Dr. Kurt Gottmann, Lehrstuhl für Zellphysiologie, Ruhr-Universität Bochum, D-44780 Bochum, Germany. E-mail: kurt@cphys.ruhr-uni-bochum.de.

Copyright (C) 1999 Society for Neuroscience 0270-6474/99/1910004-10\$05.00/0
}

in part caused by an apparently twofold reduction in mean quantal amplitude, as shown by recording AMPA receptormediated miniature PSCs. In addition, a reduced number of glutamatergic release sites in E16 target neurons was revealed by synapsin I immunostaining of dendritic presynaptic terminals. No differences in mean release probability were observed between E16 and E19 target neurons.

Thus, the formation of glutamatergic transmitter release sites was strongly influenced by target neuron-specific factors. The formation of functional GABAergic synapses, however, was independent of the type of target neurons, suggesting specific retrograde signaling during the establishment of glutamatergic synapses.

Key words: synapse formation; target neurons; glutamatergic synapses; GABAergic synapses; AMPA receptors; NMDA receptors; neocortical neurons
Davis et al., 1997; Shishido et al., 1998), and the relative balance of several recognition molecules on the target muscle appears to underlie selective synapse formation (Winberg et al., 1998). At the vertebrate neuromuscular junction, evidence has been obtained for a inductive role of retrograde signals from the postsynaptic muscle cell in the initial stages of synaptogenesis, suggesting the involvement of target recognition processes (for review, see Dan and Poo, 1994).

In the mammalian CNS, neuronal networks show an extremely high complexity of synaptic connections, which impedes the analysis of the role of target recognition in synaptogenesis. Moreover, the complex axonal arborization of central neurons makes it difficult to separate target recognition from axonal guidance processes. To reduce the complexity of the system, quantitative analysis of synapse formation in cultured dissociated neurons appears as a promising approach (Fletcher et al., 1991; Basarsky et al., 1994; Craig et al., 1994; Gottmann et al., 1994; Benson and Cohen, 1996). However, only a few studies have addressed target recognition processes in cultured central neurons. Morphological analysis of the behavior of dendritic filopodia during synaptogenesis revealed an active role of postsynaptic structures in initiating synaptogenic contacts (Morest, 1969; Ziv and Smith, 1996). Evidence for a role of the postsynaptic target in regulating the ultrastructural differentiation of presynaptic terminals has been obtained by Campbell and Frost (1987) in an in vivo study, in which the projection of retinal ganglion cell axons was experimentally altered. As shown by immunostaining for the synaptic vesicle-associated protein synapsin I, the formation of presynaptic terminals strongly depended on the state of differentiation of the postsynaptic target cells in cultured hippocampal neurons 
(Fletcher et al., 1994). Based on morphological data, these studies proposed an involvement of target neuron-derived factors in regulating the formation of presynaptic terminals.

In the present study, we have addressed the role of target neuron-specific factors in regulating synapse formation using electrophysiological techniques to quantitatively analyze the development of functional synaptic transmission and to distinguish between glutamatergic and GABAergic synapses. We used a coculture system of neocortical neurons that consisted of presynaptic explants and spatially separated, dissociated postsynaptic target neurons (Gottmann et al., 1997; Werner et al., 1998). In these cocultures, neocortical explants show a pronounced outgrowth of fibers, which innervate the dissociated neocortical target neurons that are added at low density. To assess the role of target-specific factors, we varied the type of target neurons by either using dissociated neurons obtained from the neocortex of rat embryos at embryonic day 16 (E16 target neurons) or using neocortical neurons obtained at embryonic day 19 (E19 target neurons). In contrast, the presynaptic explants were invariantly taken from embryos at embryonic day 19. Comparison of the development of glutamatergic and GABAergic synaptic transmission in both coculture systems revealed that target neurons selectively influence the formation of functional glutamatergic synapses. Our detailed electrophysiological and immunocytochemical analysis further demonstrated that the formation of glutamatergic transmitter release sites is regulated retrogradely by target neuron-specific factors.

\section{MATERIALS AND METHODS}

Cell culture. Cocultures of neocortical explants and dissociated neocortical target neurons were obtained and cultured as described (Gottmann et al., 1997; Werner et al., 1998). In brief, the occipital neocortex was removed from Wistar rat embryos at E19 and at E16, respectively. Dissociated target neurons were obtained by mechanically dissociating the neocortical tissue after trypsin $(0.1 \%)$ treatment. A $200 \mu$ ldrop of culture medium containing 3-5 $\times 10^{4}$ cells was placed in the center of a polyornithine-coated culture dish. Neocortical explants were made by cutting the occipital neocortex from E19 embryos into tissue blocks of 0.5-0.8 mm diameter. After attachment of dissociated target neurons, one E19 explant was added per culture dish. Cocultures were incubated at $37^{\circ} \mathrm{C}$ in $5 \% \mathrm{CO}_{2}$ atmosphere. The culture medium consisted of Eagle's basal medium with addition of fetal bovine serum (10\%), L-glutamine (2 $\mathrm{mM})$, glucose $(20 \mathrm{~mm})$, and insulin $(6.5 \mu \mathrm{M})$. At $5 \mathrm{~d}$ in vitro (DIV) cytosine- $\beta$-D-arabinof uranoside hydrochloride $(10 \mu \mathrm{M})$ was added to minimize the proliferation of non-neuronal cells. The origin of postsynaptic target neurons was confirmed by selectively staining the explants with DiI ( $40 \mu \mathrm{g} / \mathrm{ml} ; 12 \mathrm{hr}$ ) before cultivation. Whereas fibers growing out from the explants and innervating the dissociated target neurons were strongly stained, all target neurons that were located $>100 \mu \mathrm{m}$ outside the explant were unstained. This demonstrates that neurons do not migrate out of the explants for $>100 \mu \mathrm{m}$ under our culture conditions. Furthermore, we did not observe any differences in fiber outgrowth from explants between cocultures containing E19 or E16 target neurons.

Electrophysiology and data analysis. Somatic whole-cell voltage-clamp recordings were obtained from dissociated target neurons at room temperature using an EPC-7 patch-clamp amplifier (Heka, Lambrecht, Germany). The patch pipette solution contained (in $\mathrm{mM}$ ) $110 \mathrm{KCl}, 0.25$ $\mathrm{CaCl}_{2}, 10$ EGTA, 5 QX-314, and $20 \mathrm{HEPES}, \mathrm{pH}$ 7.3. For visualizing target neurons, Lucifer yellow $(0.1 \mathrm{mg} / \mathrm{ml})$ was added to the pipette solution. Whole-cell membrane capacitance was calculated by integrating capacitative current transients evoked by small hyperpolarizing pulses. Patch pipettes had resistances of 3-8 $\mathrm{M} \Omega$, and series resistance was compensated maximally by $50 \%$. The standard extracellular solution contained (in mM) $130 \mathrm{NaCl}, 5 \mathrm{KCl}, 5 \mathrm{CaCl}_{2}, 1 \mathrm{MgCl}_{2}$, and $20 \mathrm{HEPES}$, $\mathrm{pH}$ 7.3. To record evoked PSCs, maximal extracellular stimulation of presynaptic neurons was performed using a tungsten electrode located in the explant. Stimulation frequency was $0.1 \mathrm{~Hz}$, and stimulation strength was gradually increased until no further increase in the amplitude of evoked PCSs was observed. With excitatory synaptic transmission in the explants, undisturbed maximal stimulation resulted in bursts of AMPA PSCs lasting several hundred milliseconds that were in addition to monosynaptic activation evoked by polysynaptic activation. To quantify the development of functional synapses, monosynaptic PSC components had to be isolated. This was achieved by blocking synaptic transmission in the explants with addition of DNQX $(10 \mu \mathrm{M})$, D-AP-5 $(50 \mu \mathrm{M})$ and bicuculline methochloride $(20 \mu \mathrm{M})$ to the bath solution. Monosynaptic activation was enabled by local superfusion of the recorded target neuron (Gottmann et al., 1997) with antagonist-free extracellular solution. Blockade of excitatory synaptic transmission in the explants resulted in blockade of polysynaptic transmission, as indicated by AMPA PSCs lasting $<50$ msec. AMPA PSCs were recorded with addition of D-AP-5 and bicuculline methochloride, NMDA PSCs in $\mathrm{Mg}^{2+}$-free extracellular solution with addition of glycine $(10 \mu \mathrm{M})$, DNQX, and bicuculline methochloride, and GABA $A$ PSCs with addition of DNQX and D-AP-5 at a holding potential of $-60 \mathrm{mV}$. Mean latencies of AMPA-, NMDA-, and $\mathrm{GABA}_{\mathrm{A}}$ PSCs were $5.4 \pm 0.5,5.4 \pm 0.4$, and $4.9 \pm 0.3 \mathrm{msec}$, respectively. To verify that the vast majority of glutamatergic synapses on the postsynaptic target neuron was activated by the maximal stimulation protocol used, NMDA PSCs were evoked by maximal stimulation, and synaptic NMDA receptors were irreversibly blocked by stimulation in the presence of MK-801 $(100 \mu \mathrm{M})$. After blocking synaptic NMDA receptors in this manner, no additional NMDA receptor-mediated synaptic currents could be evoked by superfusion of the recorded neuron with high (30 $\mathrm{mM}$ ) potassium solution.

AMPA and NMDA miniature PSCs were recorded under identical conditions, except for the addition of tetrodotoxin (TTX; $1 \mu \mathrm{M}$ ). Fast whole-cell application of kainate was performed as described (Gottmann et al., 1997). PSCs were filtered on-line at $3 \mathrm{kHz}$ and sampled using pClamp 6.0 (Axon Instruments, Foster City, CA) at $30 \mathrm{kHz}$ (AMPA and $\mathrm{GABA}_{\mathrm{A}}$ PSCs) or at $4 \mathrm{kHz}$ (NMDA PSCs). Amplitudes of evoked PSCs were analyzed after averaging 10 individual PSCs using pClamp 6.0. Analysis of miniature PSC frequencies and amplitudes was performed using AUTESP software (H. Zucker, Max-Planck-Institute for Psychiatry, Munich, Germany), as described (Gottmann et al., 1994). To test the performance of our detection algorithm (amplitude threshold technique), we superimposed simulated, scaled synaptic events on electrophysiological noise and analyzed these simulated data sets. For amplitudes $\geq 6 \times$ the SD of the recording noise, $>90 \%$ of the synaptic events were detected (also see Clements and Bekkers, 1997). Because the mean $\mathrm{SD}$ of our recording noise was $1.72 \pm 0.03 \mathrm{pA}$, this means that synaptic events $\geq 10 \mathrm{pA}$ were accurately detected. PSC kinetics were analyzed using pClamp 6.0. All data are given as mean \pm SEM, and statistical analysis was done using Student's $t$ test, except for histograms that were statistically compared using the Kolmogorov-Smirnov test.

Immunocytochemistry. Presynaptic terminals were stained immunocytochemically using polyclonal Synapsin I-antibodies that were purchased from Chemicon (Temecula, CA). Synapsin I-immunostaining was performed according to Fletcher et al. (1991), as described (Gottmann et al., 1994). Brightly fluorescent puncta on dendrites representing presynaptic terminals were counted for a defined length of dendrite, and the number of presynaptic terminals per $10 \mu \mathrm{m}$ dendrite was calculated. GAD immunocytochemistry was performed with a mouse monoclonal GAD-65 antibody using the same protocol as for synapsin I-immunostaining.

\section{RESULTS}

\section{In vitro development of glutamatergic and GABAergic synaptic transmission}

The morphological differentiation of E19 and E16 target neurons in vitro did not show any significant differences, as shown by Lucifer yellow filling $(n=30)$ of target neurons (Fig. $1 C)$ and by measuring whole-cell membrane capacitances (Fig. 1D). Furthermore, the outgrowth of fibers from the presynaptic explants in our coculture system was independent of the presence of postsynaptic target neurons (Fig. 1 $A, B$ ). To compare the development of functional synapses in cocultures containing either E19 target neurons or E16 target neurons, we studied AMPA receptor-, NMDA receptor-, and $\mathrm{GABA}_{\mathrm{A}}$ receptor-mediated PSCs that were evoked by maximal extracellular stimulation in the presynaptic explant, using whole-cell patch-clamp recording at $-60 \mathrm{mV}$ holding potential. 


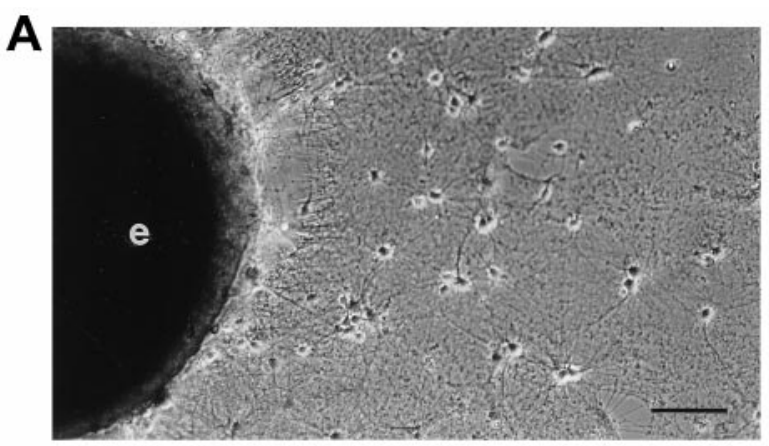

B

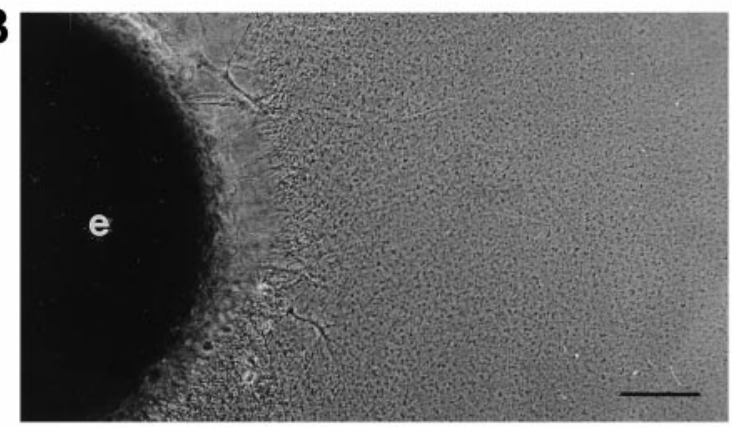

tion of 1 . Morphological differentiaaptic explants and dissociated postsynaptic target neurons. A, Photomicrograph of cocultured neocortical neurons at 12 DIV. Fibers growing out of the presynaptic explant $(e)$ innervate dissociated target neurons (obtained at E19). $B$, Without addition of dissociated target neurons, fiber outgrowth from the presynaptic explant $(e)$ is unaltered (12 DIV). Note the absence of target neurons at distances $>100 \mu \mathrm{m}$ from the explant. Scale bars: $A, B, 100 \mu \mathrm{m}$. $C$, In vitro dendritic differentiation of dissociated target neurons (9 DIV) that were obtained at E16 $(a)$ and at E19 $(b)$. Cells were filled with Lucifer yellow. Scale bars, $20 \mu \mathrm{m}$. $D$, Mean membrane capacitance of postsynaptic target neurons at different stages in culture. No differences were observed between E16 target neurons (hatched bars) and E19 target neurons (filled bars). $n$ is indicated above bars. a

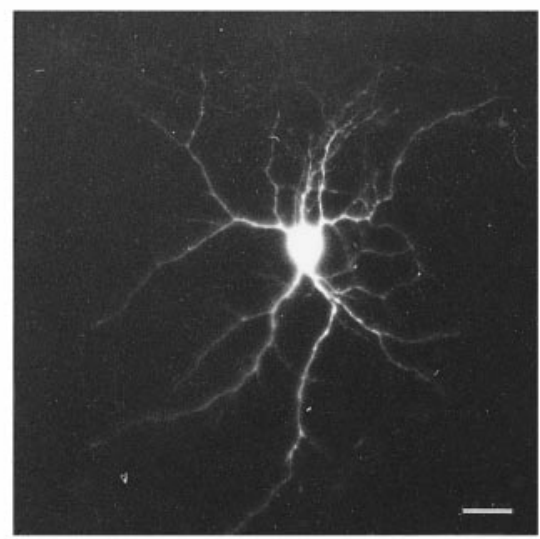

D

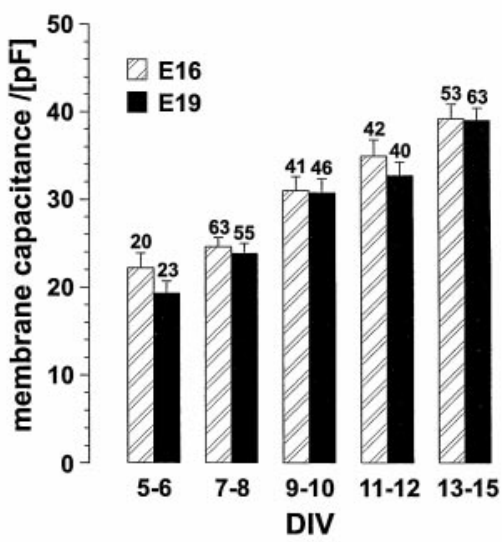

b

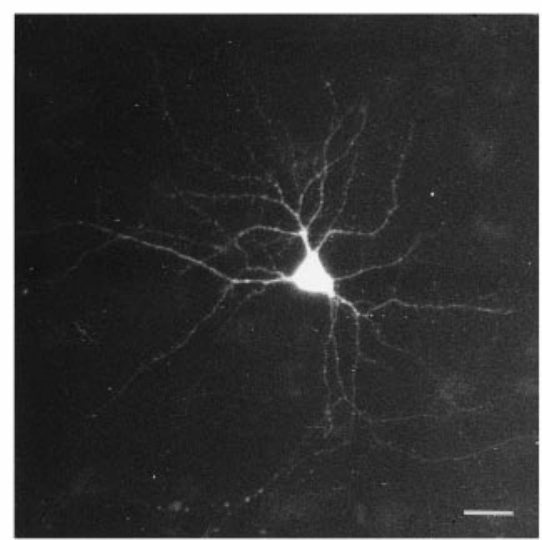

Monosynaptic AMPA receptor-mediated PSCs (AMPA PSCs) were isolated by blocking synaptic transmission in the explants with addition of DNQX (10 $\mu \mathrm{M})$, D-AP-5 (50 $\mu \mathrm{M})$, and biculline methochloride $(20 \mu \mathrm{M})$ to the bath solution and by locally superfusing the recorded target neuron with DNQX-free extracellular solution. In E19 target neurons, the mean peak amplitude of AMPA PSCs significantly $(p<0.01)$ increased from $54.0 \pm 15.6$ $\mathrm{pA}$ at 5-6 DIV to $518.0 \pm 80.7 \mathrm{pA}$ at $13-15$ DIV (Fig. $2 A$ ). Strikingly, the mean peak amplitudes of AMPA PSCs in E16 target neurons were significantly $(p<0.01)$ smaller at all stages of in vitro development (5-6 DIV, $1.8 \pm 1.2 \mathrm{pA}$; 13-15 DIV, $83.4 \pm 33.5$ pA; Fig. $2 A$ ). AMPA PSCs were reversibly blocked by addition of $10 \mu \mathrm{M}$ DNQX ( $>95 \%$ block; $n=5$; data not shown).

Pharmacologically isolated NMDA receptor-mediated PSCs (NMDA PSCs), which were recorded in $\mathrm{Mg}^{2+}$-free extracellular solution in the presence of $10 \mu \mathrm{M}$ glycine, showed similar differences in mean peak amplitudes between E19 and E16 target neurons (Fig. 2B). In E19 target neurons, the mean peak amplitude of NMDA PSCs significantly $(p<0.01)$ increased from
$11.0 \pm 7.2 \mathrm{pA}$ at $5-6$ DIV to $675.5 \pm 87.5 \mathrm{pA}$ at $13-15$ DIV. Similar to AMPA PSCs, the mean peak amplitudes of NMDA PSCs were significantly smaller $(p<0.02)$ at all stages of in vitro development in E16 target neurons (5-6 DIV, not detectable; 13-15 DIV, $116.8 \pm 28.7$ pA). NMDA PSCs were reversibly blocked by $50 \mu \mathrm{M}$ D-AP-5 (>90\% block; $n=5$; data not shown).

$\mathrm{GABA}_{\mathrm{A}}$ receptor-mediated PSCs $\left(\mathrm{GABA}_{\mathrm{A}}\right.$ PSCs) were isolated pharmacologically and recorded using symmetrical intracellular and extracellular $\mathrm{Cl}^{-}$concentrations. Most interestingly, the mean peak amplitudes of $\mathrm{GABA}_{\mathrm{A}}$ PSCs did not significantly differ between E19 and E16 target neurons at all stages of in vitro development (Fig. 2C). In E19 target neurons, the mean peak amplitude of $\mathrm{GABA}_{\mathrm{A}}$ PSCs increased significantly $(p<0.01)$ from $206.6 \pm 60.8 \mathrm{pA}$ at $5-6 \mathrm{DIV}$ to $1519.9 \pm 131.8 \mathrm{pA}$ at $13-15$ DIV. Similarly, the mean peak amplitude of GABA $_{\mathrm{A}}$ PSCs increased significantly $(p<0.01)$ from $429.3 \pm 181.2 \mathrm{pA}$ at $5-6$ DIV to $1223.7 \pm 91.0 \mathrm{pA}$ at $13-15$ DIV in E16 target neurons. $\mathrm{GABA}_{\mathrm{A}}$ PSCs were reversibly blocked by $20 \mu \mathrm{M}$ bicuculline methochloride ( $>95 \%$ block; $n=5$; data not shown). In sum- 

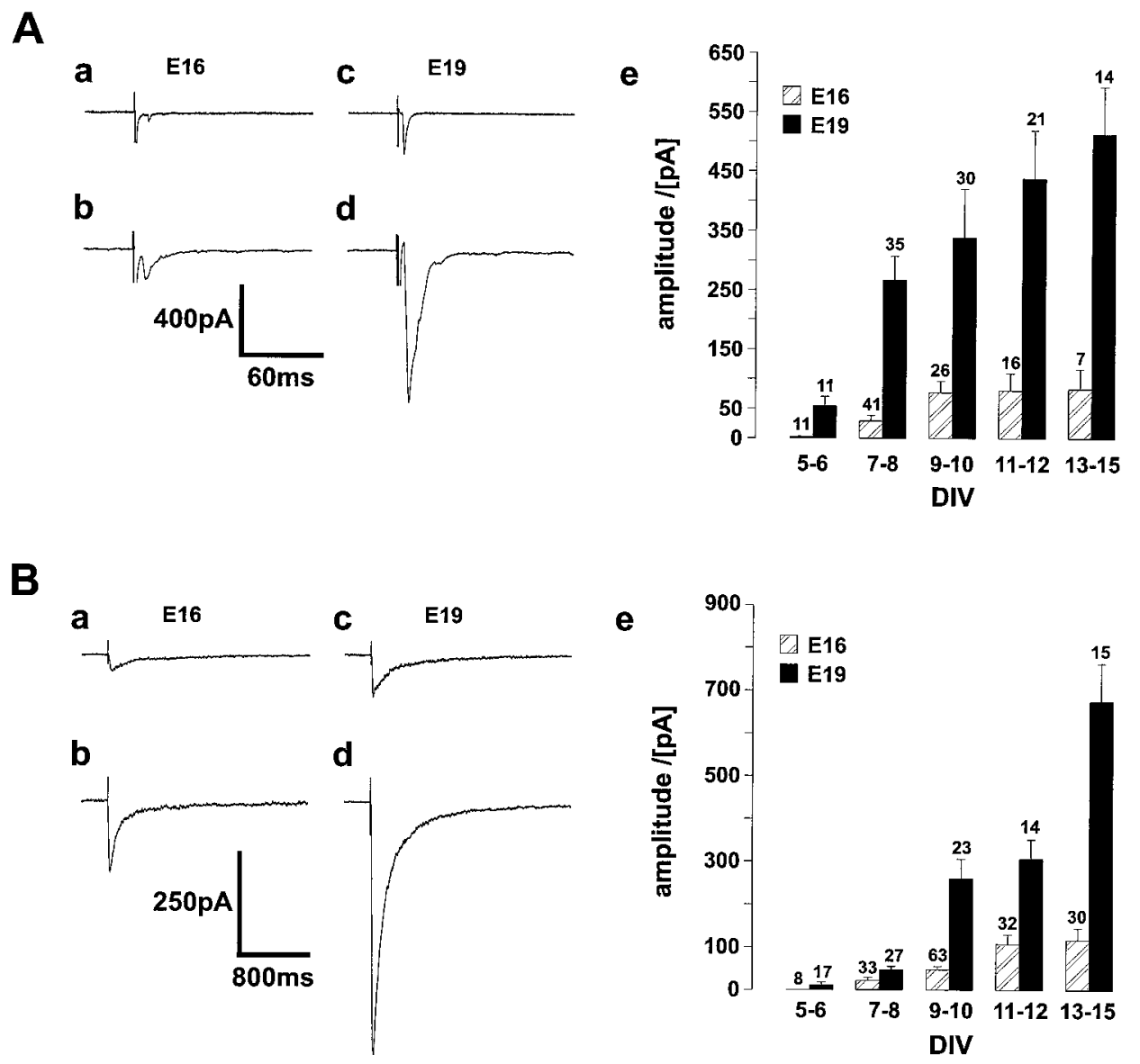

Figure 2. In vitro development of the mean amplitudes of AMPA, NMDA, and $\mathrm{GABA}_{\mathrm{A}}$ PSCs that were evoked by maximal stimulation. $A$, Monosynaptic AMPA PSCs (five individual traces averaged) evoked by maximal extracellular stimulation in the presynaptic explants in E16 target neurons at $8(a)$ and at $15(b)$ DIV and in E19 target neurons at $8(c)$ and at $15(d)$ DIV. Mean peak amplitudes of AMPA PSCs at different stages in culture are shown in $e$. E16 target neurons, hatched bars; E19 target neurons, filled bars. $n$ is indicated above bars. B, Monosynaptic NMDA PSCs (five traces averaged) evoked by maximal extracellular stimula-

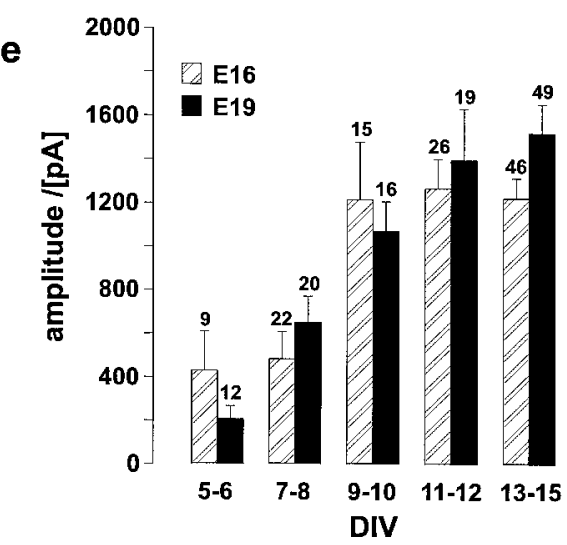
tion in the presynaptic explants in E16 target neurons at $8(a)$ and at $15(b)$ DIV and in E19 target neurons at $8(c)$ and at $15(d)$ DIV. Mean peak amplitudes of NMDA PSCs at different stages in culture are shown in $e$. $C$, Monosynaptic $\mathrm{GABA}_{\mathrm{A}}$ PSCs (five traces averaged) evoked by maximal extracellular stimulation in the presynaptic explants in E16 target neurons at 8 (a) and at 15(b) DIV and in E19 target neurons at $8(c)$ and at $15(d)$ DIV. Mean peak amplitudes of $\mathrm{GABA}_{\mathrm{A}}$ PSCs at different stages in culture are shown in $e$. Note the selective reduction in the mean peak amplitudes of AMPA and NMDA PSCs in E16 target neurons.

mary, these results demonstrate a selective inhibition of the development of functional glutamatergic synaptic transmission in cocultures containing E16 target neurons compared to cocultures containing E19 target neurons. The development of functional GABAergic synaptic transmission, however, was independent of the type of target neurons.

\section{Changes in quantal amplitude and in the number of release sites underlie reduced glutamatergic synaptic transmission in cocultures containing E16 neurons}

The observed selective reduction in the mean amplitudes of evoked AMPA and NMDA PSCs in cocultures containing E16 target neurons could be explained by changes in the quantal amplitude, in the release probability, and/or in the number of functional release sites at glutamatergic synapses. To estimate the quantal amplitude in cocultures containing E19 or E16 target neurons, we recorded pharmacologically isolated miniature AMPA PSCs (mAMPA PSCs) in the presence of $1 \mu \mathrm{M}$ TTX at $-60 \mathrm{mV}$ holding potential. Because target neurons in our coculture system do not form autapses and innervate each other with extremely low incidence because of the low cell density used (Gottmann et al., 1997), the vast majority of mAMPA PSCs can be considered to arise from synapses between explant fibers and target neurons. In E19 target neurons, the mean frequency of mAMPA PSCs significantly $(p<0.01)$ increased from $1.00 \pm$ $0.20 \mathrm{sec}^{-1}$ at $6-9$ DIV to $2.20 \pm 0.34 \mathrm{sec}^{-1}$ at $13-15$ DIV (Fig. $3 A, D)$. Amplitude histograms of mAMPA PSCs were skewed and 
A

a

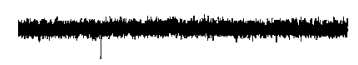

b
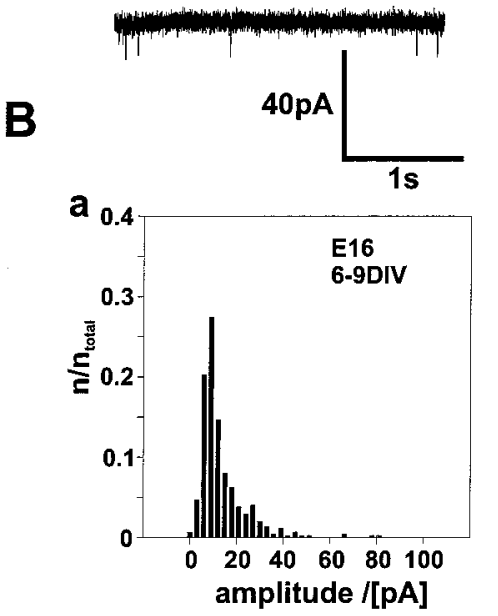

$b_{0}$

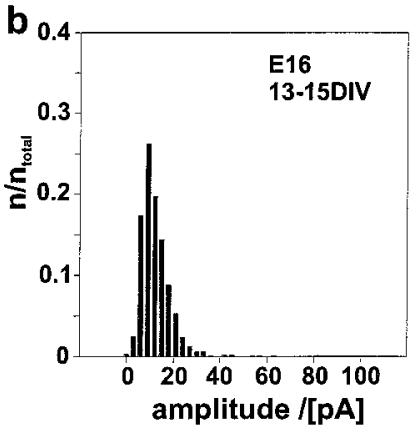

c

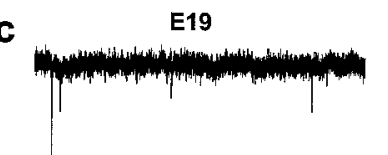

d
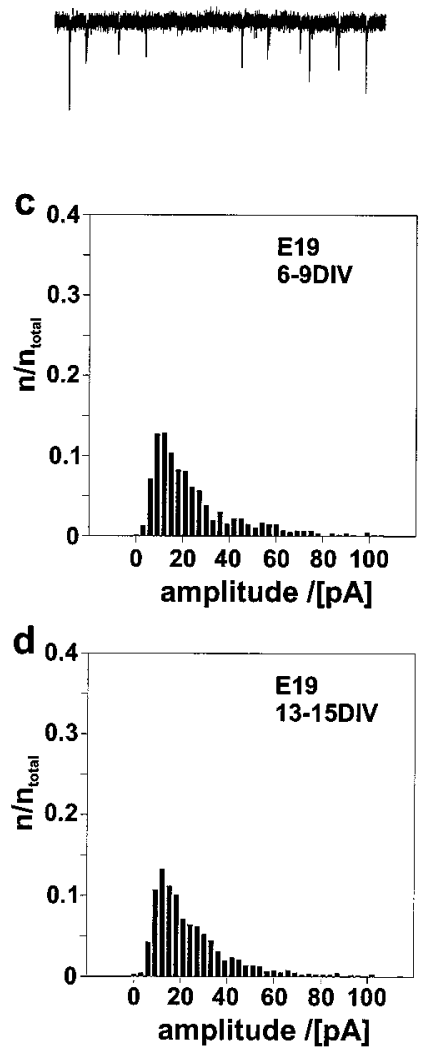

C

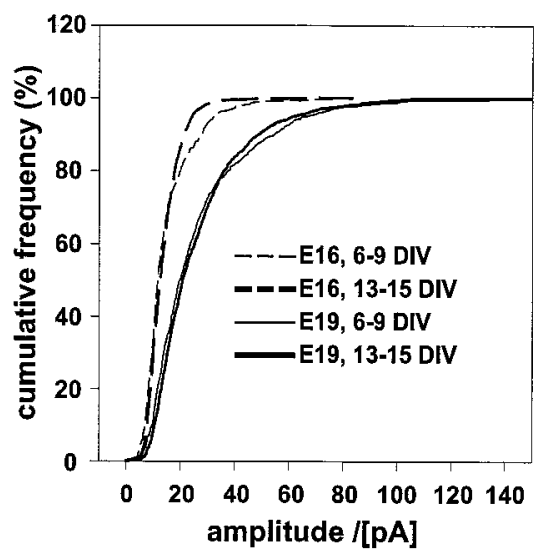

D

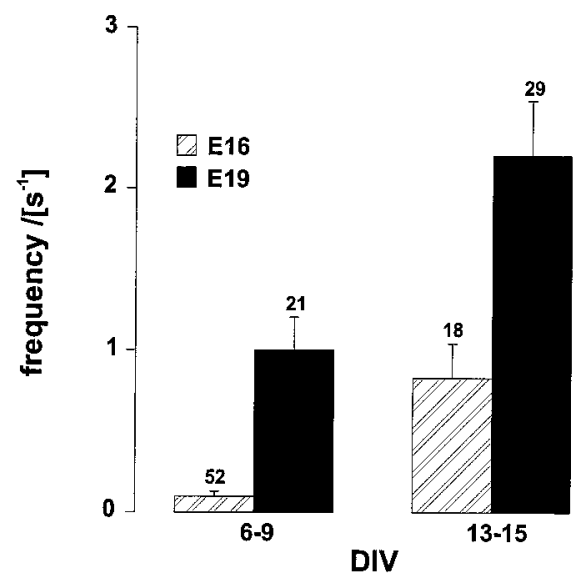

Figure 3. In vitro development of mAMPA PSCs in E16 and E19 target neurons. $A$, Examples of mAMPA PSCs that were recorded in E16 target neurons at $8(a)$ and at $15(b)$ DIV and in E19 target neurons at $8(c)$ and at $15(d)$ DIV. $B$, Amplitude distributions of mAMPA PSCs in E16 target neurons at 6-9 $(a)$ and at 13-15 (b) DIV and in E19 target neurons at 6-9 $(c)$ and at 13-15 (d) DIV. We analyzed 25-80 successive events per cell, and data from 15-30 cells were pooled. Mean mAMPA PSC amplitude was twofold larger in E19 target neurons. $C$, Cumulative distribution of mAMPA PSC amplitudes. D, Mean frequencies of mAMPA PSCs in E16 target neurons (hatched bars) and in E19 target neurons ( filled bars) at 6-9 and at 13-15 DIV. $n$ is indicated above bars.

did not significantly differ (Kolmogorov-Smirnov (KS) test; $p>$ 0.01 ) between 6-9 DIV and 13-15 DIV (Fig. 3B,C). In E19 target neurons, the mean amplitude of mAMPA PSCs was $26.5 \mathrm{pA}$ at 13-15 DIV. In E16 target neurons, the mean frequency of mAMPA PSCs was significantly $(p<0.01)$ lower at both stages analyzed and also significantly $(p<0.01)$ increased from $0.10 \pm$ $0.03 \mathrm{sec}^{-1}$ at $6-9$ DIV to $0.83 \pm 0.21 \mathrm{sec}^{-1}$ at $13-15$ DIV (Fig. $3 A, D)$. Again, amplitude histograms were skewed and did not significantly (KS test) differ between 6-9 DIV and 13-15 DIV. However, the amplitude distributions of mAMPA PSCs in E16 target neurons significantly (KS test; $p<0.01$ ) differed from those in E19 target neurons (Fig. 3B,C). In E16 target neurons, the mean amplitude of mAMPA PSCs was $13.9 \mathrm{pA}$ at 13-15 DIV and thus was twofold reduced compared to E19 target neurons. The decay kinetics of mAMPA PSCs were slightly $(p<0.05)$ slower in E19 target neurons $(\tau=3.36 \pm 0.15 \mathrm{msec})$ compared to E16 target neurons $(\tau=2.85 \pm 0.14 \mathrm{msec})$, whereas rise times did not significantly differ. Considering charge flow instead of miniature current amplitude, this results in a slightly larger (2.2-fold) difference in quantal size. However, it may be argued that a considerable proportion of mAMPA PSCs cannot be detected against the recording noise because of their small amplitude (Clements and Bekkers, 1997). This could potentially lead to an underestimation of the difference in quantal amplitude between E19 and E16 target neurons.

mAMPA PSCs were completely and reversibly blocked by 10 $\mu \mathrm{M}$ DNQX ( $n=5$; data not shown). To confirm that changes in the expression or properties of postsynaptic AMPA receptors underlie the reduced mean amplitude of mAMPA PSCs in E16 target neurons, we recorded AMPA receptor-mediated wholecell currents that were evoked by fast application of $500 \mu \mathrm{M}$ kainate. Their current density was significantly $(p<0.01)$ reduced in E16 target neurons at both stages analyzed (5-6 DIV: $\mathrm{E} 19,59.5 \pm 5.9 \mathrm{pA} / \mathrm{pF}, n=17 ; \mathrm{E} 16,10.4 \pm 2.8 \mathrm{pA} / \mathrm{pF}, n=10$; 13-15 DIV: E19, $58.8 \pm 8.0 \mathrm{pA} / \mathrm{pF}, n=14 ; \mathrm{E} 16,21.4 \pm 4.6$ $\mathrm{pA} / \mathrm{pF}, n=9$; data not shown). To this end, our results suggest a twofold reduction of the mean quantal amplitude in E16 target neurons that can only partly explain the sixfold reduction in the mean amplitude of evoked AMPA PSCs. This indicates that also the mean number of quanta contributing to AMPA PSCs might be reduced in cocultures containing E16 target neurons compared to cocultures containing E19 target neurons. 
A

a

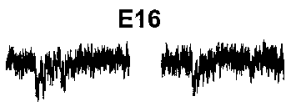

b

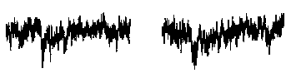

B

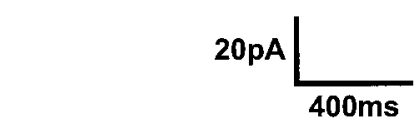

a

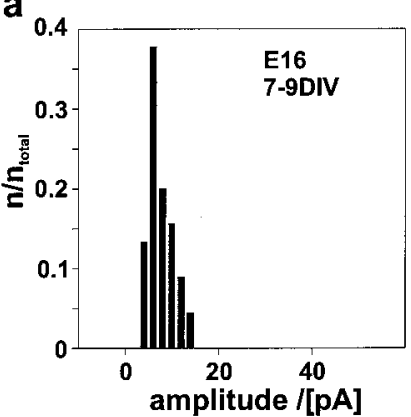

b

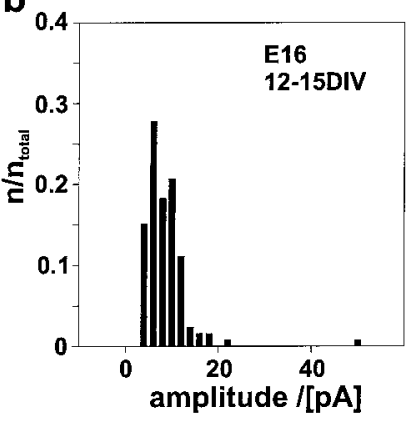

C

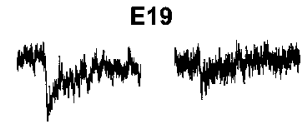

d
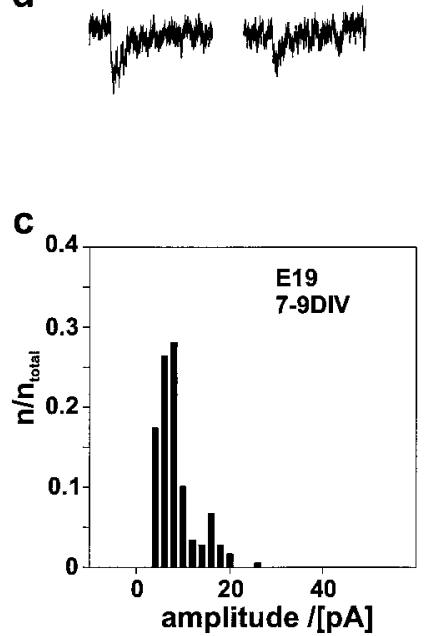

$\mathbf{d}_{0.4}$

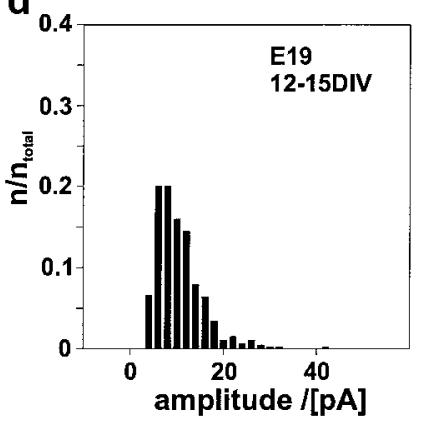

C

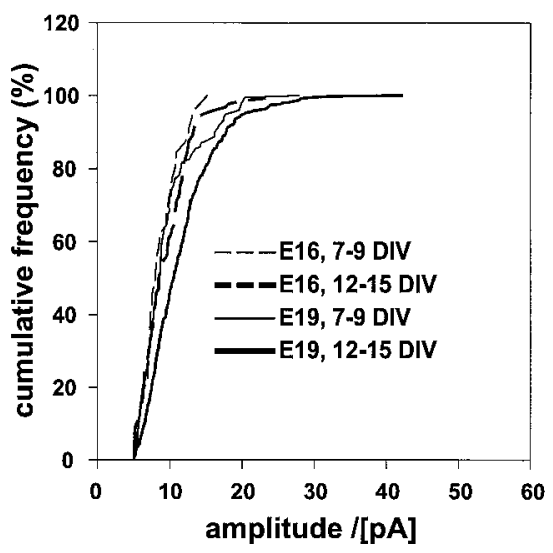

D

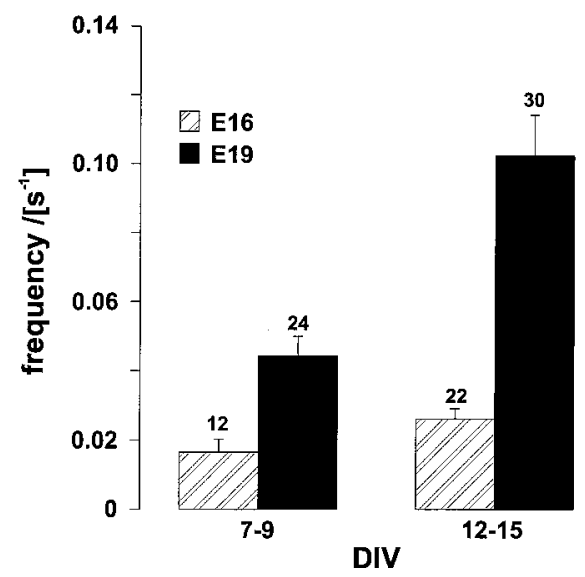

Figure 4. In vitro development of mNMDA PSCs in E16 and E19 target neurons. A, Examples of mNMDA PSCs that were recorded in E16 target neurons at $8(a)$ and at $15(b)$ DIV and in E19 target neurons at $8(c)$ and at $15(d)$ DIV. B, Amplitude distributions of mNMDA PSCs in E16 target neurons at 7-9 $(a)$ and at 12-15 (b) DIV and in E19 target neurons at 7-9 (c) and at 12-15 (d) DIV. We analyzed 10-20 events per cell, and data from 12-30 cells were pooled. A 5 pA amplitude threshold was used for detection of mNMDA PSCs. $C$, Cumulative distribution of mNMDA PSC amplitudes. $D$, Mean frequencies of mNMDA PSCs in E16 target neurons (hatched bars) and in E19 target neurons (filled bars) at 7-9 and at 12-15 DIV. $n$ is indicated above bars.

In addition to mAMPA PSCs, we also recorded pharmacologically isolated miniature NMDA PSCs (mNMDA PSCs) in cocultures containing E19 or E16 target neurons in the presence of $1 \mu \mathrm{M}$ TTX in nominally $\mathrm{Mg}^{2+}$-free extracellular solution containing $10 \mu \mathrm{M}$ glycine at a holding potential of $-60 \mathrm{mV}$. In E19 target neurons, the mean frequency of mNMDA PSCs significantly $(p<0.01)$ increased from $0.044 \pm 0.006 \mathrm{sec}^{-1}$ at $7-9$ DIV to $0.102 \pm 0.012 \mathrm{sec}^{-1}$ at $12-15 \mathrm{DIV}$ (Fig. $4 D$ ). In E16 target neurons, the mean frequency of mNMDA PSCs was significantly $(p<0.01)$ lower at both stages analyzed and slightly $(p=0.06)$ increased from $0.017 \pm 0.004 \mathrm{sec}^{-1}$ at $7-9$ DIV to $0.026 \pm 0.003$ $\mathrm{sec}^{-1}$ at $12-15$ DIV (Fig. 4D). Although it has been shown that AMPA and NMDA receptors are colocalized at the majority of glutamatergic synapses in cultured cortical neurons (Bekkers and Stevens, 1989), the detected mNMDA PSC frequencies were considerably lower than mAMPA PSC frequencies in both coculture systems. However, because evoked AMPA and NMDA PSCs showed comparable amplitudes, this difference could be explained by technical difficulties in detecting mNMDA PSCs that are caused by the increased background noise in $\mathrm{Mg}^{2+}$-free solution and by the slow time course of mNMDA PSCs. The amplitude distributions of mNMDA PSCs did not significantly differ between E19 target neurons and E16 target neurons at both stages analyzed (KS test; $p>0.01$ ), although mNMDA PSCs in E19 target neurons tended to be larger (Fig. 4B,C). mNMDA PSCs were reversibly blocked by $50 \mu \mathrm{M}$ D-AP-5 ( $n=5$; data not shown). Similar to the results obtained from the analysis of AMPA PSCs, these results suggest that the observed reduction in the mean amplitude of evoked NMDA PSCs in E16 target neurons arises at least in part from a reduced mean number of quanta of evoked NMDA PSCs.

We next addressed whether differences in mean release probability between cocultures containing E19 or E16 target neurons can account for the observed reduction in the mean amplitudes of evoked AMPA and NMDA PSCs. We compared release probabilities at glutamatergic synapses in E19 and E16 target neurons by investigating the kinetics of the use-dependent blockade of NMDA PSCs by the irreversible NMDA receptor antagonist MK-801. Based on the colocalization of AMPA and NMDA receptors at glutamatergic synapses (Bekkers and Stevens, 1989), this method is well established to give a reliable relative measure of the mean release probability of a population of glutamatergic 
A

a

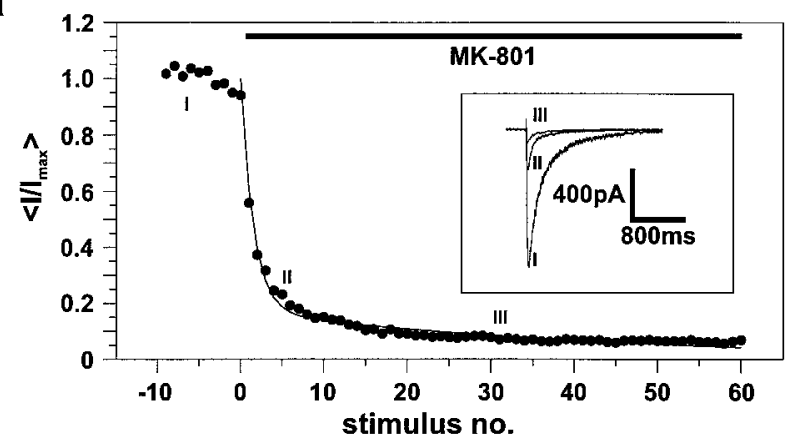

B

a

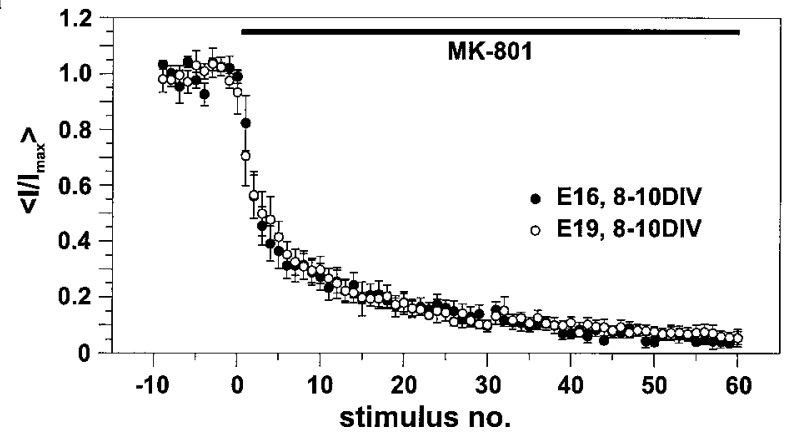

b

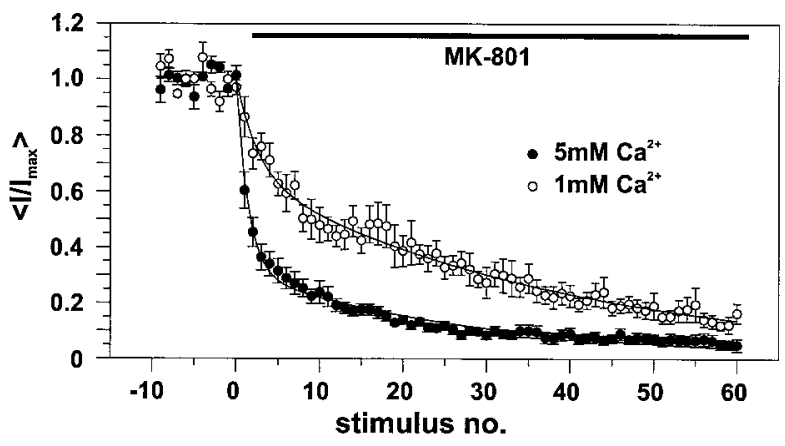

b

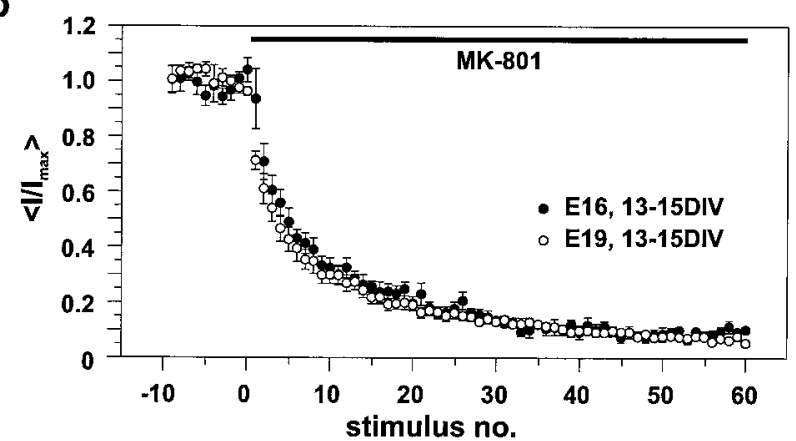

Figure 5. Estimation of the mean release probability by the progressive block of NMDA PSCs by MK-801. A, Use-dependent block of NMDA PSCs by $20 \mu \mathrm{M}$ MK-801 in an E19 target neuron at 14 DIV $(a)$. The progressive decay of NMDA PSC peak amplitude was fitted by a biexponential function. Inset shows NMDA PSCs at indicated time points. Lowering the extracellular $\mathrm{Ca}^{2+}$ concentration from 5 ( filled dots) to 1 (empty dots) mM resulted in a significantly slowed MK-801 block of NMDA PSCs in E19 target neurons at 9-13 DIV $(b)$. $B$, The kinetics of MK-801 block of NMDA PSCs did not differ between E16 target neurons ( filled dots) and E19 target neurons (empty dots) at 8-10 $(a)$ and at 13-15 (b) DIV. Fit parameters were: 8-10 DIV, E19 $(n=9): \tau_{\text {fast }} 2.23 \pm 0.85$ stimuli, $\tau_{\text {slow }} 29.01 \pm 5.44$ stimuli, $\mathrm{A}_{\text {fast }} /\left(\mathrm{A}_{\text {fast }}+\mathrm{A}_{\text {slow }}\right) 0.62 \pm 0.07 ; \mathrm{E} 16(n=9): \tau_{\text {fast }} 2.23 \pm 0.41$ stimuli, $\tau_{\text {slow }} 35.42 \pm 6.38$ stimuli, $\mathrm{A}_{\text {fast }} /\left(\mathrm{A}_{\text {fast }}+\mathrm{A}_{\text {slow }}\right) 0.62 \pm 0.09 ; 13-15 \mathrm{DIV}, \mathrm{E} 19(n=8): \tau_{\text {fast }} 2.59 \pm 0.06$ stimuli, $\tau_{\text {slow }} 29.36 \pm 3.02$ stimuli, $\mathrm{A}_{\text {fast }} /\left(\mathrm{A}_{\text {fast }}+\mathrm{A}_{\text {slow }}\right) 0.58 \pm 0.06$; $\mathrm{E} 16(n=8): \tau_{\text {fast }} 4.38 \pm 0.92$ stimuli, $\tau_{\text {slow }} 46.81 \pm 8.62$ stimuli, $\mathrm{A}_{\text {fast }} /\left(\mathrm{A}_{\text {fast }}+\mathrm{A}_{\text {slow }}\right) 0.66 \pm 0.07$.

synapses (Hessler et al., 1993; Rosenmund et al., 1993; Manabe and Nicoll, 1994; Huang and Stevens, 1997).

In E19 target neurons at 9-13 DIV, addition of $20 \mu \mathrm{M}$ MK-801 led to a progressive block of evoked NMDA PSCs with increasing number of stimulations that could be well fitted with a doubleexponential function with mean time constants of $2.0 \pm 0.7$ stimuli and $32.7 \pm 7.9$ stimuli (Fig. $5 A$ ). To confirm that the used method is sensitive to changes in the release probability, we reduced the extracellular $\mathrm{Ca}^{2+}$ concentration from 5 to $1 \mathrm{~mm}$. In $1 \mathrm{mM} \mathrm{Ca}^{2+}$, the progressive block of NMDA PSCs by MK-801 was clearly slowed (Fig. $5 A$ ). The mean time constants did not significantly change $\left(1 \mathrm{mM} \mathrm{Ca}^{2+}: 2.8 \pm 0.9\right.$ stimuli and $39.4 \pm 4.6$ stimuli), whereas the contribution of the fast decaying component was significantly $(p<0.05)$ reduced from $0.61 \pm 0.07$ in $5 \mathrm{~mm}$ $\mathrm{Ca}^{2+}(n=8)$ to $0.32 \pm 0.09$ in $1 \mathrm{mM} \mathrm{Ca}^{2+}(n=6)$. However, we did not observe any significant differences in the kinetics of MK-801 block of NMDA PSCs between E19 and E16 target neurons at both stages analyzed (Fig. $5 B$ ). These results strongly suggest that no differences in mean release probability occur between cocultures containing E19 and E16 target neurons, and thus changes in release probability cannot account for the observed reduced mean amplitudes of evoked AMPA and NMDA PSCs in E16 target neurons. Because the observed differences in mean quantal amplitudes can only partly explain the differences in the mean amplitudes of evoked AMPA and NMDA PSCs, our results suggest that also the number of functional glutamatergic release sites is reduced in E16 target neurons.

A reduction in the number of functional glutamatergic release sites in E16 target neurons could be explained by an increased incidence of postsynaptically silent glutamatergic synapses (Liao et al., 1995; Durand et al., 1996; Isaac et al., 1997; Kiyosue et al., 1997; Rumpel et al., 1998), that show exclusively NMDA receptor-mediated synaptic transmission. However, in E16 target neurons the mean amplitudes of evoked NMDA PSCs and the frequency of mNMDA PSCs were also clearly reduced, thus excluding the above possibility. An increased incidence of presynaptically silent synapses (Kimura et al., 1997) or a reduced number of glutamatergic presynaptic terminals in E16 target neurons could also account for a reduction in the number of functional glutamatergic release sites.

\section{The density of dendritic presynaptic terminals is reduced in E16 target neurons}

To study the development of presynaptic terminals in cocultures containing E19 or E16 target neurons, presynaptic terminals were immunostained using polyclonal synapsin I antibodies at 7 and 14 DIV. Staining with synapsin I antibodies resulted in bright fluorescent puncta outlining the dendrites (Fig. 6), which have previously been shown in cultured cortical neurons to represent presynaptic terminals (DeCamilli et al., 1983; Fletcher et al., 

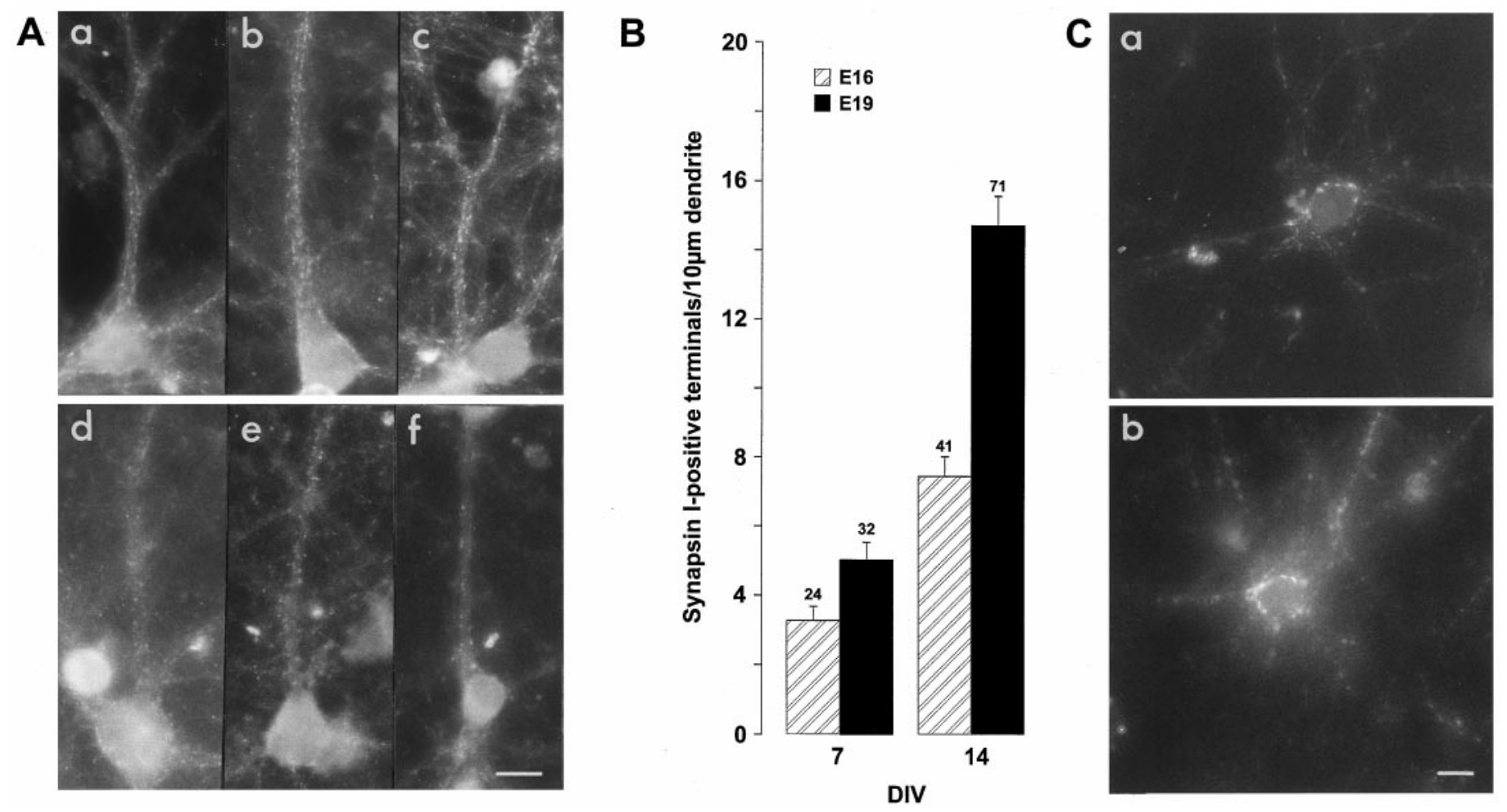

Figure 6. Synapsin I and GAD-65 immunostaining of presynaptic terminals in E16 and E19 target neurons. $A$, Examples of dendritic presynaptic terminals immunostained with a polyclonal synapsin I antibody in E19 target neurons $(a-c)$ and in E16 target neurons $(d-f)$ at 14 DIV. Scale bar, $10 \mu \mathrm{m}$. $B$, Mean number of synapsin I-immunopositive terminals per $10 \mu \mathrm{m}$ dendrite length in E16 target neurons (hatched bars) and in E19 target neurons ( filled bars) at 7 and at 14 DIV. $n$ is indicated above bars. The mean number of presynaptic terminals was significantly reduced in E16 target neurons at both stages. $C$, Somatic presynaptic terminals immunostained with a GAD-65 antibody in an E19 target neuron $(a)$ and in an E16 target neuron $(b)$. Dendritic GAD-65-positive presynaptic terminals are partly out of focus. Scale bar, $10 \mu \mathrm{m}$.

1991). To quantify the number of presynaptic terminals in E19 and E16 target neurons, we counted the number of bright fluorescent puncta on a defined length of dendrite. In E19 target neurons, the mean number of synapsin I-positive terminals per 10 $\mu \mathrm{m}$ dendrite increased significantly $(p<0.01)$ from $5.0 \pm 0.5$ at 7 DIV to $14.7 \pm 0.8$ at 14 DIV. In E16 target neurons, the mean number of synapsin I-positive terminals per $10 \mu \mathrm{m}$ dendrite was significantly $(p<0.01)$ lower at both stages analyzed and also increased during in vitro development from $3.3 \pm 0.4$ at 7 DIV to $7.4 \pm 0.6$ at 14 DIV.

To address the formation of GABAergic presynaptic terminals in cocultures containing E19 or E16 target neurons, we immunostained GABAergic presynaptic terminals at 14 DIV using a mouse monoclonal GAD-65 antibody. Staining with the GAD-65 antibody led to bright fluorescent puncta located on the soma and on the dendrites of target neurons (Fig. 6). To quantitatively compare the formation of GABAergic presynaptic terminals in E19 and E16 target neurons, we again counted the number of puncta on the soma and on a defined length of dendrite. The mean number of GAD-65-positive terminals on the soma did not significantly differ between E19 (10.7 \pm 1.2) and E16 target neurons $(11.8 \pm 1.0)$. Similarly, the mean number of GAD-65positive terminals per $10 \mu \mathrm{m}$ dendrite did not significantly differ between E19 (3.1 \pm 0.4$)$ and E16 target neurons (3.6 \pm 0.3$)$. In line with our observations, it has been demonstrated in cultured hippocampal neurons that the majority of dendritic presynaptic terminals is glutamatergic (Benson and Cohen, 1996).

In summary, our results demonstrate a reduced number of presynaptic terminals in E16 target neurons compared to E19 target neurons. Because the mean amplitudes of evoked $\mathrm{GABA}_{\mathrm{A}}$ PSCs and the mean number of GABAergic presynaptic terminals were not different between E19 and E16 target neurons, the above results strongly suggest a selective reduction in the number of glutamatergic presynaptic terminals in E16 target neurons.

\section{DISCUSSION}

In this study we have analyzed the importance of target neuronspecific factors in the development of functional synaptic transmission in cultured rat neocortical neurons. To selectively alter the properties of postsynaptic target neurons, we used dissociated neurons that were obtained at different stages of neocortical development (either at E16 or at E19) as target neurons and cocultured them with presynaptic explants obtained from E19 embryos. At E16, the cortical plate has not yet formed, and neocortical cells are starting to migrate from the ventricular zone to their final positions. In contrast, at E19 the cortical plate is well developed, and the neurons of the deep cortical layers have already reached their final positions (Kageyama and Robertson, 1993). Thus, dissociated neurons from E19 embryos contain a considerable proportion of postmigratory neurons, which are not present at E16. Postmigratory neurons appear to strongly differ from migratory neurons regarding the expression of cell surface proteins that mediate cell adhesion (Götz et al., 1992; McConnell, 1995; Tuttle et al., 1995).

Several earlier studies have demonstrated that the growth of thalamocortical and corticocortical fibers into neocortical tissue or on neocortical membranes is strongly dependent on the stage of differentiation at which explants or membranes were obtained 
(Götz et al., 1992; Barbe and Levitt, 1995; Tuttle et al., 1995). These studies proposed a maturation-dependent upregulation of growth-promoting molecules in neocortical tissue that strongly influences axonal guidance processes. Because of the special design of our coculture system, i.e., a large neocortical explant that interacts with a relatively small number of dissociated neurons, we did not observe any influence of the presence and type of postsynaptic target neurons on the outgrowth of fibers from the explants. Therefore, our cocultures allow us to directly study target cell recognition processes during synaptogenesis, without indirect effects on synapse formation that are primarily caused by alterations in axon growth.

Our detailed electrophysiological analysis of in vitro synaptogenesis in cocultures containing either E19 or E16 target neurons suggested a retrograde regulation of the formation of functional glutamatergic transmitter release sites by target neuron-specific factors. The immunocytochemical analysis of the number of presynaptic terminals also revealed a regulation of the formation of presynaptic terminals by target neuron-specific factors that selectively influenced the formation of glutamatergic synapses, whereas GABAergic synapses were not affected.

Using maximal extracellular stimulation in the presynaptic explant to quantitatively activate all synapses on the recorded postsynaptic target neuron, we observed a strong reduction of the mean peak amplitudes of evoked AMPA and NMDA PSCs in cocultures containing E16 target neurons. With activation of a large number of synapses, transmitter release tended to be asynchrounous, thus leading to an underestimation of the mean peak amplitudes of AMPA PSCs in E19 target neurons at late stages in culture. Based on quantal transmitter release, the selective reduction in the mean amplitudes of AMPA and NMDA PSCs can be explained by changes in the quantal amplitude and/or in the number of quanta released (Redman, 1990). To investigate the contribution of changes in the quantal amplitude, we analyzed spontaneous miniature PSCs, which represent the postsynaptic response to the spontaneous release of single quanta (Stevens, 1993; Forti et al., 1997; Frerking et al., 1997). As typical of miniature PSCs in cultured cortical neurons, their amplitude distributions were skewed, most likely reflecting the presence of multiple synapses and dendritic filtering (Bekkers et al., 1990; Bekkers and Stevens, 1995; Forti et al., 1997). The observed reduction of quantal amplitudes in E16 target neurons can in part cause the reduction in mean amplitudes of evoked AMPA and NMDA PSCs. However, to fully account for the observed strong reduction in the mean amplitudes of evoked AMPA and NMDA PSCs, a reduction in the mean number of quanta released has to be assumed in addition. By comparing the kinetics of MK-801 block of NMDA PSCs between the two coculture systems, we found no significant differences in mean release probability. Thus, our electrophysiological data suggest that the number of functional glutamatergic release sites is reduced in E16 target neurons.

A reduction in the number of functional glutamatergic release sites could be explained either by a reduced formation of presynaptic terminals or by an increased incidence of nonfunctional synapses that do not release glutamate. Our immunocytochemical results strongly suggest that a reduced number of glutamatergic presynaptic terminals underlies the reduction in the number of functional release sites. An increased incidence of presynaptically silent synapses, which show presynaptic accumulation of synaptic vesicles but do not release transmitter (Kimura et al., 1997), may also contribute to the reduction in the number of functional release sites. In summary, our results demonstrate a strong dependence of the formation of glutamatergic transmitter release sites on properties of the postsynaptic target neuron, thus suggesting a retrograde regulation of the formation of glutamatergic presynaptic terminals. Based on morphological data, a retrograde regulation of the formation of presynaptic terminals has previously also been suggested in cultured hippocampal neurons (Fletcher et al., 1994), however, no distinction between glutamatergic and GABAergic terminals was made in that study.

The molecular mechanisms underlying the retrograde regulation of the formation of glutamatergic release sites remain to be elucidated. Target recognition processes involving membrane surface proteins like cell adhesion molecules might play a major role. Candidate molecules include cadherins (Fannon and Colman, 1996; Uchida et al., 1996), densin-180 (Apperson et al., 1996), neuroligins (Irie et al., 1997; Song et al., 1999), and members of the Ig superfamily (Lüthi et al., 1994). Interestingly, maturationdependent changes in the expression of cell adhesion molecules have been described in differentiating neocortical neurons (Götz et al., 1992; Tuttle et al., 1995). Alternatively, mechanisms involving the postsynaptic secretion of trophic factors like neurotrophins appear conceivable (Wang and Poo, 1997; VicarioAbejon et al., 1998). Trophic factors might play a major role in regulating neuronal differentiation, including the surface expression of adhesion molecules, in neocortical neurons.

Moreover, our observation that the development of GABAergic synaptic transmission was independent of the type of target neurons, whereas the development of glutamatergic synapses was heavily affected, strongly indicates that different molecular mechanisms regulate the formation of glutamatergic and GABAergic presynaptic terminals in neocortical neurons. Consistent with this notion, the expression of $\mathrm{N}$-cadherin strikingly differs between glutamatergic and GABAergic synapses in cultured hippocampal neurons (Benson and Tanaka, 1998). Furthermore, a selective clustering of the receptor anchoring protein gephyrin at GABAergic but not at glutamatergic synapses has been described in cultured hippocampal neurons (Craig et al., 1996).

In the developing mammalian neocortex synaptogenesis starts in deep layers and occurs in an inside-out pattern that parallels the inside-out gradient of neuronal differentiation (Blue and Parnavelas, 1983; Chun and Shatz, 1988). Below the differentiating cortical plate developmentally transient functional synapses are formed by thalamocortical afferents in the subplate region (Friauf et al., 1990). The observed inability of immature neocortical neurons to retrogradely induce the formation of glutamatergic presynaptic terminals may play a major role in the developmental regulation of the spatial pattern of synapse formation in the neocortex.

\section{REFERENCES}

Apperson ML, Moon IS, Kennedy MB (1996) Characterization of densin-180, a new brain-specific synaptic protein of the Osialoglycoprotein family. J Neurosci 16:6839-6852.

Barbe MF, Levitt P (1995) Age-dependent specification of the corticocortical connections of cerebral grafts. J Neurosci 15:1819-1834.

Basarsky TA, Parpura V, Haydon PG (1994) Hippocampal synaptogenesis in cell culture: developmental time course of synapse formation, calcium influx, and synaptic protein distribution. J Neurosci 14:6402-6411.

Bekkers JM, Stevens CF (1989) NMDA and non-NMDA receptors are co-localized at individual excitatory synapses in cultured rat hippocampus. Nature 341:230-233.

Bekkers JM, Stevens CF (1995) Quantal analysis of EPSCs recorded from small numbers of synapses in hippocampal cultures. J Neurophysiol 73:1145-1156. 
Bekkers JM, Richerson GB, Stevens CF (1990) Origin of variability in quantal size in cultured hippocampal neurons and hippocampal slices. Proc Natl Acad Sci USA 87:5359-5362.

Benson DL, Cohen PA (1996) Activity-independent segregation of excitatory and inhibitory synaptic terminals in cultured hippocampal neurons. J Neurosci 16:6424-6432.

Benson DL, Tanaka H (1998) N-cadherin redistribution during synaptogenesis in hippocampal neurons. J Neurosci 18:6892-6904.

Blue ME, Parnavelas JG (1983) The formation and maturation of synapses in the visual cortex of rat. II. Quantitative analysis. J Neurocytol 12:697-712.

Campbell G, Frost DO (1987) Target-controlled differentiation of axon terminals and synaptic organization. Proc Natl Acad Sci USA 84:6929-6933.

Chiba A, Snow P, Keshishian H, Hotta Y (1995) Fasciclin III as a synaptic target recognition molecule in Drosophila. Nature 374:166-168.

Chun JJM, Shatz CJ (1988) Redistribution of synaptic vesicle antigens is correlated with the disappearance of a transient synaptic zone in the developing cerebral cortex. Neuron 1:297-310.

Clements JD, Bekkers JM (1997) Detection of spontaneous synaptic events with an optimally scaled template. Biophys J 73:220-229.

Craig AM, Blackstone CD, Huganir RL, Banker G (1994) Selective clustering of glutamate and $\gamma$-aminobutyric acid receptors opposite terminals releasing the corresponding neurotransmitters. Proc Natl Acad Sci USA 91:12373-12377.

Craig AM, Banker G, Chang W, McGrath ME, Serpinskaya AS (1996) Clustering of gephyrin at GABAergic but not glutamatergic synapses in cultured rat hippocampal neurons. J Neurosci 16:3166-3177.

Dan Y, Poo M (1994) Retrograde interactions during formation and elimination of neuromuscular synapses. Curr Opin Neurobiol 4:95-100.

Davis GW, Schuster CM, Goodman CS (1997) Genetic analysis of the mechanisms controlling target selection: target-derived Fascilin II regulates the pattern of synapse formation. Neuron 19:561-573.

DeCamilli P, Harris SM, Huttner WB, Greengard P (1983) Synapsin I (protein I), a nerve terminal-specific phosphoprotein. II. Its specific association with synaptic vesicles demonstrated by immunocytochemistry in agarose-embedded synaptosomes. J Cell Biol 96:1355-1373.

Durand GM, Kovalchuk Y, Konnerth A (1996) Long-term potentiation and functional synapse induction in developing hippocampus. Nature 381:71-75.

Fannon AM, Colman DR (1996) A model for central synaptic junctional complex formation based on the differential adhesive specificities of the cadherins. Neuron 17:423-434.

Fletcher TL, Cameron P, DeCamilli P, Banker G (1991) The distribution of synapsin I and synaptophysin in hippocampal neurons developing in culture. J Neurosci 11:1617-1626.

Fletcher TL, DeCamilli P, Banker G (1994) Synaptogenesis in hippocampal cultures: evidence indicating that axons and dendrites become competent to form synapses at different stages of neuronal development. J Neurosci 14:6695-6706.

Forti L, Bossi M, Bergamaschi A, Villa A, Malgaroli A (1997) Loosepatch recordings of single quanta at individual hippocampal synapses. Nature 388:874-878.

Frerking M, Borges S, Wilson M (1997) Are some minis multiquantal? J Neurophysiol 78:1293-1304.

Friauf E, McConnell SK, Shatz CJ (1990) Functional synaptic circuits in the subplate during fetal and early postnatal development of cat visual cortex. J Neurosci 10:2601-2613.

Götz M, Novak N, Bastmeyer M, Bolz J (1992) Membrane-bound molecules in rat cerebral cortex regulate thalamic innervation. Development 116:507-519.

Goodman CS, Shatz CJ (1993) Developmental mechanisms that generate precise patterns of neuronal connectivity. Cell 72/Neuron 10 [Suppl]:77-98.

Gottmann K, Pfrieger FW, Lux HD (1994) The formation of glutamatergic synapses in cultured central neurons: selective increase in miniature synaptic currents. Dev Brain Res 81:77-88.

Gottmann K, Mehrle A, Gisselmann G, Hatt H (1997) Presynaptic control of subunit composition of NMDA receptors mediating synaptic plasticity. J Neurosci 17:2766-2774.

Huang EP, Stevens CF (1997) Estimating the distribution of synaptic reliabilities. J Neurophysiol 78:2870-2880.
Hessler NA, Shirke AM, Malinow R (1993) The probability of transmitter release at a mammalian central synapse. Nature 366:569-572.

Irie M, Hata Y, Takeuchi M, Ichtchenko K, Toyoda A, Hirao K, Takai Y, Rosahl TW, Südhof TC (1997) Binding of neuroligins to PSD-95. Science 277:1511-1515.

Isaac JTR, Crair MC, Nicoll RA, Malenka RC (1997) Silent synapses during development of thalamocortical inputs. Neuron 18:269-280.

Kageyama GH, Robertson RT (1993) Development of geniculocortical projections to visual cortex in rat: evidence for early ingrowth and synaptogenesis. J Comp Neurol 335:123-148.

Keshishian H, Broadie K, Chiba A, Bate M (1996) The Drosophila neuromuscular junction: a model system for studying synaptic development and function. Annu Rev Neurosci 19:545-575.

Kimura F, Otsu Y, Tsumoto T (1997) Presynaptically silent synapses: spontaneously active terminals without stimulus-evoked release demonstrated in cortical autapses. J Neurophysiol 77:2805-2815.

Kiyosue K, Kasai M, Taguchi T (1997) Selective formation of silent synapses on immature postsynaptic cells in cocultures of chick neurons of different ages. Dev Brain Res 99:201-207.

Liao D, Hessler NA, Malinow R (1995) Activation of postsynaptically silent synapses during pairing-induced LTP in CA1 region of hippocampal slice. Nature 375:400-404.

Lüthi A, Laurent J-P, Figurov A, Muller D, Schachner M (1994) Hippocampal long-term potentiation and neural cell adhesion molecules L1 and NCAM. Nature 372:777-779.

Manabe T, Nicoll RA (1994) Long-term potentiation: evidence against an increase in transmitter release probability in the CA1 region of the hippocampus. Science 265:1888-1892.

McConnell SK (1995) Plasticity and commitment in the developing cerebral cortex. Prog Brain Res 105:129-143.

Morest DK (1969) The growth of dendrites in the mammalian brain. Z Anat Entwicklungsgesch 128:290-317.

Redman S (1990) Quantal analysis of synaptic potentials in neurons of the central nervous system. Physiol Rev 70:165-198.

Rosenmund C, Clements JD, Westbrook GL (1993) Nonuniform probability of glutamate release at a hippocampal synapse. Science 262:754-757.

Rumpel S, Hatt H, Gottmann K (1998) Silent synapses in the developing rat visual cortex: evidence for postsynaptic expression of synaptic plasticity. J Neurosci 18:8863-8874.

Shishido E, Takeichi M, Nose A (1998) Drosophila synapse formation: regulation by transmembrane protein with Leu-rich repeats, Capricious. Science 280:2118-2121.

Song, JY, Ichtchenko K, Südhof TC, Brose N (1999) Neuroligin 1 is a postsynaptic cell-adhesion molecule of excitatory synapses. Proc Natl Acad Sci USA 96:1100-1105.

Stevens CF (1993) Quantal release of neurotransmitter and long-term potentiation. Cell 72/Neuron 10 [Suppl]:55-63.

Tessier-Lavigne M, Goodman CS (1996) The molecular biology of axon guidance. Science 274:1123-1133.

Tuttle R, Schlaggar BL, Braisted JE, O‘Leary DM (1995) Maturationdependent upregulation of growth-promoting molecules in developing cortical plate controls thalamic and cortical neurite growth. J Neurosci 15:3039-3052.

Uchida N, Honjo Y, Johnson KR, Wheelock MJ, Takeichi M (1996) The catenin/cadherin adhesion system is localized in synaptic junctions bordering transmitter release zones. J Cell Biol 135:767-779.

Vicario-Abejon C, Collin C, McKay RDG, Segal M (1998) Neurotrophins induce formation of functional excitatory and inhibitory synapses between cultured hippocampal neurons. J Neurosci 18:7256-7271.

Wang X, Poo M (1997) Potentiation of developing synapses by postsynaptic release of neurotrophin-4. Neuron 19:825-835.

Werner M, Hatt H, Gottmann K (1998) Afferent innervation influences $\mathrm{HVA} \mathrm{Ca}^{2+}$ current expression in cultured neocortical neurones. NeuroReport 9:1255-1260.

Winberg ML, Mitchell KJ, Goodman CS (1998) Genetic analysis of the mechanisms controlling target selection: complementary and combinatorial functions of netrins, semaphorins, and IgCAMs. Cell 93:581-591.

Ziv NE, Smith SJ (1996) Evidence for a role of dendritic filopodia in synaptogenesis and spine formation. Neuron 17:91-102. 